\title{
Le juste prix des produits alimentaires : entre efficience des marchés et exigence de justice sociale
}

Patrice Guillotreau

\section{(2) OpenEdition \\ Journals}

Édition électronique

URL : http://journals.openedition.org/economierurale/3960

DOI : $10.4000 /$ economierurale.3960

ISSN : 2105-2581

Éditeur

Société Française d'Économie Rurale (SFER)

Édition imprimée

Date de publication : 15 mai 2013

Pagination : 87-94

ISSN : 0013-0559

\section{Référence électronique}

Patrice Guillotreau, «Le juste prix des produits alimentaires : entre efficience des marchés et exigence de justice sociale », Économie rurale [En ligne], 335 | mai-juin 2013, mis en ligne le 15 mai 2015, consulté le 19 avril 2019. URL : http://journals.openedition.org/economierurale/3960 ; DOI : 10.4000/ economierurale.3960 


\title{
ÉTAT DE L'ART
}

\section{Le juste prix des produits alimentaires Entre efficience des marchés et exigence de justice sociale}

\author{
Patrice GUILLOTREAU • LEMNA, Université de Nantes
}

Les prix jugés trop bas des produits agricoles soulèvent périodiquement la question du juste prix. La notion de juste prix est débattue depuis l'origine même des sciences économiques mais tend à se confondre davantage, dans son acception moderne, avec l'efficience des marchés - au sens de justesse des prix - qu'avec l'exigence de justice sociale introduite par Rawls. Ce bref article vise à rappeler l'évolution du concept depuis Aristote jusqu'à sa formalisation néoclassique de plus en plus contestée. La justice commutative aristotélicienne semble ainsi réhabilitée dans les travaux récents sur l'équilibre de justice (fairness equilibrium) indispensable au bon déroulement des échanges. Mais les transformations de l'organisation marchande, telles que les cotations électroniques ou encore le recours à la finance, ne risquent-elles pas de perturber la recherche du juste prix ?

\section{Le constat de la baisse des prix}

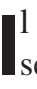
est bien difficile de comptabiliser l'ensemble des secteurs alimentaires (fruits, lait, produits de la mer...) où les producteurs se sentent insuffisamment rémunérés pour leurs productions et dénoncent des prix jugés trop faibles, en particulier en 2009, après le déclenchement de la crise financière internationale. Certains chiffres produits par l'INSEE sont éloquents : baisse de $16 \%$ du prix moyen des produits agricoles entre juillet 2008 et juillet 2009 (-32\% pour les céréales, $-34 \%$ pour les fruits, $-24 \%$ pour le lait, $-19 \%$ pour les légumes, $-11 \%$ pour les volailles, $-8 \%$ pour le porc, $-6 \%$ pour le bœuf, $-3 \%$ pour les vins). La cause est bien sûr conjoncturelle et liée à la crise économique qui a frappé l'Europe, mais les tendances de long terme affichent une baisse tendancielle du prix des matières premières agricoles.

Sur longue période, la tendance structurelle montre en effet une dégradation des termes de l'échange. Le prix du caoutchouc a été divisé par trois entre 1960 et 1990 et par vingt au cours du vingtième siècle. Certains calculs de Prebisch et Singer effectués dans les années 1950 ou encore les indices de prix de Grilli et Yang sur la période 1900-1986, calculés sur 18 produits (blé, maïs, huile de palme, bœuf, mouton, sucre, banane...) concluent à une baisse globale de $40 \%$ sur la période, soit un rythme annuel moyen de $-0,6 \%$ environ. Certains analystes apportent quelques nuances lorsqu'ils examinent les tendances de long terme en distinguant les périodes (Geronimi, 2012)1. Les prix des produits agroalimentaires ont baissé de manière beaucoup plus faible, voire ont été stables pendant une longue période. Seuls les produits agricoles non alimentaires ont véritablement connu cette tendance baissière de longue période. L'effet volume compense parfois l'effet de baisse des cours. Les périodes sont également contrastées. Le prix réel des produits agricoles est effectivement bas depuis le début des années 1990, mais il a augmenté de 1957 à 1973 avant de baisser de 1974 à 2004. La dernière

1. Geronimi V., Maître d'Hôtel É. (2012). Les évolutions récentes des cours des matières premières agricoles : enjeux de développement et de sécurité alimentaire. Revue Tiers-Monde, $\mathrm{n}^{\circ}$ 211, p. 7-12. 
décennie, marquée par la demande en forte croissance des pays émergents, semble inverser cette tendance. Mais si l'on retient sur longue période un constat général de tendance à la décroissance des prix, peuton parler de «prix injuste » dans le cas des produits alimentaires, en référence à la notion aristotélicienne ou scholastique du « juste prix »?

\section{Le " juste prix " lié à l'origine de la valeur des biens}

Cette notion de juste prix est très ancienne puisqu'elle remonte aux philosophes de la Grèce antique, qui réduisaient la fonction de l'économie à la seule subsistance du foyer, c'est-à-dire au maintien physiologique de la vie. Incomprise par les économistes et par Schumpeter en particulier, la théorie du juste prix chez Aristote fait intervenir deux dimensions : l'effort déployé pour se procurer le bien et le statut social du vendeur par rapport à l'acquéreur. Aristote concevait ainsi l'existence d'un « commerce naturel » dont le juste prix serait le support et favoriserait une relation de réciprocité entre les intérêts des partenaires de l'échange. Quand cette réciprocité d'intérêts est rompue, le prix ne serait plus juste. On retrouve cette notion de justice associée au prix des biens dans la philosophie scholastique du Moyen Âge, notamment dans l'œuvre de Thomas d'Aquin à propos de la distinction aristotélicienne entre justice distributive et justice commutative. Cette dernière renvoie à l'idée d' " égalité dans l'échange ». Pour Thomas d'Aquin, aucun des partenaires ne doit profiter de l'échange au détriment de l'autre. Après Adam Smith et les premiers économistes classiques (Say, Malthus, Ricardo, Sismondi), la notion de subsistance se confond avec la valeur des choses. «Le principe universel des valeurs vénales ou échangeables, c'est la subsistance; elle est l'élément primitif dans lequel elles se résolvent toutes en dernière analyse. La valeur d'un objet quelconque [...], dans la main d'un pauvre, c'est la quantité de subsistance que cet objet pourra lui procurer pour sa consommation. » (de CarrionNisas, 1825). ${ }^{2}$

Sans entrer trop profondément dans la distinction entre valeur d'usage (incommensurable par nature) et valeur d'échange, revenons à l'essence de la valeur théorisée par les premiers économistes classiques. Dans son essai sur la richesse des nations $(1776)^{3}$, Adam Smith écrit : «Le prix réel de chaque chose [...], est l'équivalent de la peine et de l'embarras qu'il a fallu pour l'acquérir. Pour l'acquéreur, c'est la peine et l'embarras que cette chose peut lui épargner, et qu'elle a le pouvoir de rejeter sur d'autres personnes. » Jean-Baptiste Say compare l'utilité d'un bien ordinaire comme le clou et celle d'une épingle d'or qui, bien que moins utile, vaut 2000 fois plus (Say, 1828) ${ }^{4}$. Que dire alors des biens libres comme l'air ou l'eau, gratuits malgré leur utilité encore plus grande ? Il en conclut que la valeur n'est pas proportionnée à l'utilité, mais à la peine 2000 fois plus grande qu'il a fallu à l'ouvrier pour se procurer l'épingle d'or comparativement à celle nécessaire à la fabrication d'un clou. «Toutes les manières de donner de l'utilité, ou d'augmenter celle que les choses ont déjà, se réduisent à l'art de leur faire subir une modification quelconque ; car nous ne créons aucune matière. Tout ce que nous pouvons faire, c'est de les transformer, réunir, diviser, mêler, changer de place, pour les rendre propres à l'usage des hommes. » (Say, 1828).

2. Carrion-Nisas A. de (1825). Principes d'économie politique. Paris, Raymond, $287 \mathrm{p}$.

3. Smith A. (1776). La richesse des nations. Paris, GF Flammarion, trad. fr. 1991.

4. Say J.-B. (1828). Cours d'économie politique et autres essais. Paris, GF-Flammarion, éd. 1996, $450 \mathrm{p}$. 
À partir de cette quantité relative de travail, les marchés convergent vers des « prix naturels », sortes d'équilibre de long terme. «Le prix naturel du travail est celui qui fournit aux ouvriers les moyens de subsister et de perpétuer leur espèce, sans accroissement ni diminution » (Ricardo, $1817)^{5}$. Il conçoit qu'une heure de travail d'un ouvrier ordinaire n'est pas équivalente à une heure de travail d'un ouvrier qualifié dans une bijouterie, mais sans expliquer vraiment cette différence. Quand il étudie les prix agricoles, il envisage bien la rémunération d'un autre facteur que le travail (la rente du propriétaire foncier), mais celle-ci n'est que la conséquence et non la cause de la valeur. Le prix va se fixer par rapport à la mauvaise terre, c'est-à-dire la moins productive et le propriétaire de la bonne terre pourra ainsi dégager une rente différentielle (ou rente de rareté) tandis que le premier aura une rente nulle. Marx ajoute à la rente de Ricardo la notion de surprofit fondée sur la distinction entre valeur (créée par l'usage du travail) et prix (qui repose sur la valeur d'échange de la force de travail). Même sa conception de la rente est différente de celle de Ricardo : elle représente le revenu du propriétaire et non un simple écart de productivité entre des terres. Pour les denrées agricoles, le prix ajoute donc à la rente ricardienne cette rente absolue (et non relative) comme le ferait un impôt (Marx, 1872) ${ }^{6}$.

La théorie néo-classique du dix-neuvième siècle remet au goût du jour la théorie de la valeur utilité fondée sur la rareté, dont Ricardo avait minimisé l'importance. Désormais, c'est du côté de la demande que se fonde la valeur des biens et la conception walrassienne s'impose alors. Selon cette conception, prix et valeur se

5. Ricardo D. (1817). Des principes de l'économie politique et de l'impôt. Paris, Champs Flammarion, éd. 1977, 379 p.

6. Marx K. (1872) Le capital. Paris, Lachatre, livre III (http://gallica.bnf.fr). confondent à nouveau. Le prix est déterminé par le fonctionnement du marché et la relation offre/demande. La science économique moderne - qui se démarque peu à peu de l'économie politique des auteurs classiques - ne se pose donc pas la question du juste prix. Ou plus exactement, elle y répond de façon détournée et procédurale par le fonctionnement même du marché. Qu'est ce qu'un « juste prix » dans le cadre d'une économie de marché, sinon celui qui découle de la confrontation entre demande agrégée et offre disponible.

\section{Spécificités des marchés agricoles et fluctuations du " juste prix »}

Demande et offre des produits agricoles obéissent à des règles spécifiques et les grandes fluctuations que subit le prix de tels biens ne peuvent surprendre quiconque s'intéresse à ces marchés. La hausse des rendements accroît tendanciellement l'offre. Rappelons qu'entre 1930 et 2000, les rendements du maïs ont été multipliés par un facteur 4 , le progrès étant de même ampleur pour les rendements laitiers (Tietenberg, 2012) 7 . Il en va ainsi de la plupart des productions agricoles. Gregory King, un généalogiste anglais, montrait dès 1696 qu'une récolte abondante de blé se traduit sur le plan économique par des revenus en baisse pour les producteurs. Cournot précisait en 1838 les conditions de validité de la Loi de King sur le prix et le revenu en longue période : une hausse de la production agricole peut engendrer une baisse des revenus en cas de faible élasticité-prix de la demande, le prix baissant plus rapidement que n'augmente la quantité produite. Les producteurs ont donc intérêt, en cas de récolte abondante, à s'organiser pour détruire une partie de la production.

7. Tietenberg T. (2012). Environmental and natural resource economics. $9^{\mathrm{e}}$ édition, Boston, Mass., Pearson, $666 \mathrm{p}$. 
Ils ne le font pas la plupart du temps et les conditions environnementales qui influencent les récoltes entretiennent la forte variabilité des cours du fait de la rigidité de l'offre au prix. Les aléas climatiques ou biologiques confèrent à ces marchés une nature stochastique qui les singularise. À cette incertitude exogène s'ajoute une instabilité structurelle et endogène liée aux effets de retard entre l'investissement et la production. Le célèbre modèle du Cobweb (Ezekiel, 1938) ${ }^{8}$ rend compte du rôle des anticipations et des retards d'ajustement de l'offre dans l'instabilité des prix. Le cycle du prix du porc est ainsi érigé en exemple dans les manuels d'économie afin de souligner les effets récurrents du sur-investissement sur les prix, les périodes d'abondance succédant aux phases de pénurie.

En vertu de cette nature endogène de l'instabilité des marchés agricoles, certains auteurs vont même jusqu'à douter de l'efficacité des instruments financiers de couverture du risque de prix (Boussard, 1996) ${ }^{9}$. Les tentatives de régulation du cours des matières premières agricoles par des accords internationaux ayant toutes plus ou moins échoué depuis les années 1950, les producteurs se tournent désormais vers les marchés dérivés pour se couvrir contre le risque de prix. Mais ces marchés peuvent s'écarter sensiblement des fondamentaux du marché physique. Ainsi, les récentes envolées du prix des céréales et du maïs en particulier $(+27 \%$ en deux mois de septembre à novembre 2009 sur le marché à terme de Chicago) défiaient toutes les prévisions des analystes constatant alors une stabilité de l'offre et un effondrement de la demande. Outre la croissance soutenue de la demande en provenance des pays émergents au cours de la décennie passée, une

8. Ezekiel M. (1938). The cobweb theorem. Quarterly Journal of Economics, vol. 52, n 2, p. 255-280. 9. Boussard J.-M. (1996). When risk generates chaos. Journal of Economic Behavior and Organization, $\mathrm{n}^{\circ} 29$, p. 433-446. explication avancée pour de telles hausses ponctuelles et brutales concernerait les liquidités massives injectées par les banques centrales pour juguler la crise et venant se placer sur les indices des matières premières agricoles pour créer de nouvelles bulles spéculatives. Les contrats à terme sur le blé «Soft Trade Winter » représentent ainsi plus de quatre fois la récolte réelle. Autre chiffre éloquent fourni par les statistiques du FMI : l'indice mensuel du prix mondial des produits alimentaires (base 100 en 2005) est passé de 95 en moyenne sur la période 1991-2005 (avec un écart-type de 10) à 145 en moyenne sur la période 2006-2012 (avec un écart-type de 25) (source : www.imf.org). Cette augmentation de moitié en valeur et en volatilité bénéficie temporairement aux producteurs, mais reflète-t-elle véritablement un changement vers un prix plus juste si l'on considère que les prix agricoles étaient jusqu'alors anormalement bas, ou une nouvelle source d'injustice à venir au détriment cette fois des demandeurs ?

Le « juste prix » issu de la loi du marché serait ainsi soumis à de grands aléas. Et d'ailleurs, de quel marché parle-t-on ? Le mode d'organisation des échanges diffère d'un produit à l'autre (prix affichés, négociation de gré à gré, contrats de long terme, enchères, marchés physiques ou virtuels, cotation électronique, etc.) et ce fait est relativement peu pris en considération par les économistes. Plusieurs études montrent cependant que des prix plus élevés peuvent être obtenus lorsqu'on substitue aux contrats de gré à gré des prix d'enchères, même si ces derniers accroissent bien souvent la variabilité des cours. Les systèmes électroniques de vente au cadran auraient un impact non négligeable sur les prix à la première vente. Le cas des langoustines vendues au Guilvinec et Lorient le prouve : la mise en place d'un système d'enchères électroniques dans les deux points de vente en mars et avril 2002, en lieu et place d'un 
système de vente à la voix et d'un système de gré à gré, a accru les prix moyens de $16 \%$ à Lorient et de $8 \%$ au Guilvinec, et les variances respectivement de 2 et 6 points (Guillotreau, Jiménez-Toribio $2011)^{10}$. La révolution Internet, qui permet désormais la vente synchronisée à distance, modifie également l'organisation de marché et produirait un effet positif sur les prix de certains produits qui connaissaient ponctuellement des niveaux de prix bas.

\section{Le " juste prix » entendu comme le produit d'un rapport social}

L'équilibre offre-demande fournissant le « juste » prix suppose, bien évidemment, que les marchés soient eux-mêmes parfaitement organisés et concurrentiels, sans distorsion aucune, pour déployer leur pleine efficacité. Mettre en relation « efficacement » offreurs et demandeurs est en soi un exercice de formatage inspiré parfois de la théorie économique elle-même (Callon, 1998 ; MacKenzie et al., 2007) ${ }^{11}$. Rejoignant la conception d'Artistote, un tel exercice traduit un compromis (i.e. une construction) résultant du rapport social entre acteurs sur le marché (Graham, 1999) $)^{12}$.

Paul Jorion suggère dans un ouvrage récent que le « juste prix » observé procède ainsi non pas du rapport direct entre offre et demande, bien que celui-ci puisse expliquer les variations du prix, mais d'un « rapport de force institutionnellement

10. Guillotreau P., Jiménez-Toribio R. (2011). The price effect of expanding fish auction markets. Journal of Economic Behavior and Organization, $\mathrm{n}^{\circ} 79$, p. 211-225.

11. Callon M. (1998). The Laws of the markets. Oxford, Blackwell Publishers, 278 p.

MacKenzie D., Muniesa F., Siu L. (dir.) (2007). Do Economists Make Markets? On the Performativity of Economics. Princeton, Princeton University Press.

12. Graham I. (1999). The construction of electronic markets. Édimbourg, Thèse de doctorat. cautionné » qu'il nomme « statut réciproque » (Jorion, 2010) ${ }^{13}$. Il en veut pour preuve le fait que les variations dans les prix sont souvent de moindre amplitude que les variations du rapport quantités offertes/quantités demandées. La bonne volonté commune des uns et des autres, apparentée à la philia aristotélicienne ${ }^{14}$, sert d'amortisseur aux désavantages trop marqués que constituerait une offre tantôt surabondante, tantôt déficitaire. Aucun des partenaires de l'échange ne chercherait ainsi à pousser trop loin son avantage pour ne pas léser le partenaire, avec qui il partage un intérêt supérieur et solidaire de long terme (pas d'offreur sans demandeur et réciproquement). Nombreux sont les cas où la loyauté d'une relation marchande, c'est-à-dire un lien durable entre deux opérateurs malgré des opportunités de marché parfois plus intéressantes pour l'un ou pour l'autre, peut paradoxalement s'accompagner chez l'acheteur de prix plus élevés que la moyenne des autres transactions (Weisbuch et al., 2000) ${ }^{15}$. L'acheteur s'acquitte ainsi d'une sorte de « prime d'assurance » destinée à sécuriser ses approvisionnements lorsque l'offre sera moins abondante car il sera alors servi en priorité par l'offreur. Mais ce rapport de réciprocité, qui vaut pour un marché organisé de gré à gré, tiendrait-il toujours sur un marché de cotation électronique lorsque l'anonymat des transactions rompt avec toute possibilité de loyauté ? Il faut donc bien considérer un autre rapport, plus constant et socialement institué, entre l'offreur et le demandeur, présentant un caractère de proportionnalité au sein d'une

13. Jorion P. (2010). Le prix. Paris, Éditions du Croquant, $364 \mathrm{p}$.

14. La philia découle du sentiment philanthropique d'appartenance à une même communauté souhaitant la réalisation du bien commun.

15. Weisbuch G., Kirman A. P., Herreiner D. (2000). Market organisation and trading relationships. The Economic Journal, n 110, p. 411-436. 
« enveloppe forfaitaire » à se partager selon le statut social des partenaires (Jorion, 2010).

Un autre fait vient étayer la thèse aristotélicienne du statut réciproque des partenaires de l'échange agissant comme une proportion : le caractère relativement universel du statut entre producteur et marchand dans des contextes aussi culturellement différents que des sociétés occidentales et africaines (ibid.). Pour stable qu'il apparaisse, ce rapport social n'en est pas pour autant immuable et peut fluctuer selon les circonstances. L'auteur cite ainsi, dans le cas d'une société médiévale, le statut des ruraux par rapport aux citadins qui ne manquerait pas de s'inverser si la ville était assiégée et en proie à la pénurie.

La structure généralement oligopsonique des marchés agricoles reflète donc davantage le rapport social entre offreurs et acheteurs dans la fixation du prix que le seul équilibre entre quantités de biens produites et consommées. «Les marchés réels sont plus souvent de type oligopsonique que concurrentiels, et la signification des prix agricoles doit aussi être recherchée du côté de la théorie de l'économie dominante » (Malassis, 1963) ${ }^{16}$. Comme l'écrit François Perroux : «Maintes circonstances conduiraient aujourd'hui à penser qu'il est aussi opportun de concevoir le monde économique comme un ensemble de rapports patents ou dissimulés entre dominants et dominés que comme un ensemble de rapports entre égaux. »

\section{Justice des prix versus efficience des marchés}

On peut donc objecter que l'on raisonne, dans la conception néo-classique, en termes d'efficience et non de justice ou d'équité qui se rapprocherait davantage

16. Malassis L. (1963). Prix agricoles et planification. Économie rurale, n ${ }^{\circ} 56$, p. 3-8. d'une théorie du contrat (Rawls, 1971) ${ }^{17}$. Cette vision confondant juste prix et efficience des marchés domine encore aujourd'hui, bien que des économistes se soient intéressés à la notion de « justice dans l'échange » dès la fin du dix-neuvième siècle (par exemple, Gustav Schmoller et l'école historique allemande). Certes, le marché reste le point de rencontre de comportements intéressés et égoïstes, mais une exigence sociale de régulation peut se manifester chaque fois qu'un sentiment d'injustice émerge en raison de l'inégalité dans laquelle se trouvent les participants. C'est ainsi que naissent des institutions comme le droit du travail ou le droit de la concurrence visant à protéger les plus faibles. Le point de vue de Schmoller est ici positif, et donc résolument différent du point de vue normatif de Thomas d'Aquin ou d'Aristote. Il vise à comprendre comment l'exigence de justice sociale va formater institutionnellement les marchés pour remédier au sentiment d'injustice (Hédoin, 2008) ${ }^{18}$. Cette idée est reprise dans des travaux récents en économie comportementale et expérimentale pour montrer comment les prix se forment à partir de points focaux au-dessus ou en dessous desquels il n'est pas moralement acceptable de descendre.

À titre d'exemple, une étude conduite par le prix Nobel Daniel Kahneman montrait que la population de Toronto avait perçu comme injuste l'élévation du prix des pelles à neige par un magasin en situation de monopole à la suite d'une importante chute de neige. L'attitude du vendeur était pourtant conforme à la théorie économique néoclassique : anticipant une forte hausse de la demande, il avait ajusté ses prix à la hausse.

17. Rawls J. (1971). Théorie de la justice, trad. fr., Paris, Seuil, chap. 1, « La justice comme équité », p. 180-202.

18. Hédoin C. (2008). Le juste prix existet-il ? Site internet Rationalité Limitée, 15 septembre 2008, http://rationalitelimitee.wordpress. com/2008/09/15/le-juste-prix-existe-t-il/ 
Mais une telle attitude n'était pas moralement convenable. De fait, les individus ne cessent de former des représentations sur ce que constitue un «niveau acceptable» de profit ou n'acceptent le principe d'une variation des prix que si elle est fondée sur la variation des coûts de l'entreprise. « D'un point de vue scientifique, les jugements que portent les agents sur ce qui est "juste" ne doivent pas être évalués normativement mais ils doivent être pris pour ce qu'ils sont : des manifestations d'une exigence de justice qui ont un impact sur les phénomènes économiques » (Hédouin, 2008). La norme peut se fonder sur des principes moraux, laïcs ou religieux. Ainsi, le producteur «ne doit pas exiger plus pour son travail et ses dépenses que ce qui lui permet de maintenir son statut [...]. Et s'il exige davantage en vue de s'enrichir ou d'améliorer son train de vie, il commet le péché d'avarice » (de Roover, 1958) $)^{19}$.

L'idée d'un « équilibre de justice » (fairness equilibrium) fait peu à peu son chemin : dans l'interaction de l'échange, l'individu intègre dans sa propre utilité l'idée qu'il se fait du jugement de l'autre sur son comportement vis-à-vis de la réciprocité. En d'autres termes, «si je pense qu'autrui s'attend à ce que je sois honnête et que je me comporte de manière effectivement honnête, alors cela augmente ma satisfaction. » (Rabin, 1993) ${ }^{20}$. On lève ainsi le comportement systématiquement non coopératif de la théorie des jeux tel qu'il est mis en évidence par le célèbre dilemme du prisonnier (Axelrod, 1984) ${ }^{21}$ pour fonder une «économie de la confiance »

19. Roover (de) R. (1958). The concept of the just price: theory and economic policy. Journal of Economic History, $\mathrm{n}^{\circ} 18$, p. 418-434.

20. Rabin M. (1993). Incorporating Fairness into Game Theory and Economics. American Economic Review, vol. 83, $\mathrm{n}^{\circ}$ 5, p. 1281-1302.

21. Axelrod R. (1984). The evolution of cooperation. New York, Basic Books.
(Laurent, 2012) ${ }^{22}$ dans laquelle les individus acquièrent une mémoire des coopérations passées (Ostrom, Walker, 2003) ${ }^{23}$.

\section{Métamorphoses du marché et reconquête du juste prix}

En raison des nouvelles formes d'organisation des marchés alimentaires (cotations électroniques, vente à distance, rôle de la finance, concentration du commerce de détail, allongement des filières, etc.), les consommateurs se voient offrir des possibilités infinies de privilégier le pouvoir d'achat au détriment des exigences de justice à l'égard des producteurs. Ils trouvent ainsi réunis dans un même point de vente des biens à bon marché, produits en masse, incorporant davantage de services (portions individuelles, emballages, facilité de préparation...) et dont l'origine importe finalement assez peu. Les producteurs répondent à ce moins-disant en trichant parfois sur les matières premières utilisées, comme le prouve le récent scandale de la viande de cheval incorporée à la place du bœuf dans les lasagnes et conserves de viande vendues en France. Producteurs et consommateurs tendent ainsi à rompre le lien social qui les unissait en raison d'un éloignement de plus en plus grand engendré par ces métamorphoses du marché. L'équilibre de justice exigé par le contrat social au sein d'une communauté d'intérêts de long terme s'en trouve fragilisé (qui se soucie de la juste rémunération des producteurs situés aux antipodes ?). Les pressions à la hausse ou à la baisse des prix agricoles proviennent de multiples facteurs qui s'avèrent difficiles à contrôler simultanément pour intégrer une norme de justice dans les rapports marchands.

22. Laurent E. (2012). Économie de la confiance. Paris, La Découverte, coll. « Repères », 124 p. 23. Ostrom E., Walker J. (dir.) (2003). Trust and reciprocity. Interdisciplinary lessons from experimental research, New York, Russell Sage Foundation. 


\section{ÉTAT DE L'ART}

Certaines initiatives récentes sur les marchés réels (vente directe, appellations d'origine, AMAP, commerce équitable...) tentent quant à elles de renouer le lien distendu entre producteurs et consommateurs et répondent ainsi à cette exigence sociale de justice dans la formation du prix à laquelle offreurs et demandeurs ne devraient rester durablement insensibles. Bien que subjective et positive, la valeur des biens alimentaires doit tenir compte de cette attente des consommateurs-citoyens. Achevons cette réflexion par une citation qui invite à un rapprochement plus étroit entre économie et justice sociale :
«Au XIX siècle, dans le cadre du capitalisme triomphant, la vie économique s'est dépouillée de toute considération de justice. [...] Le prix apparaissait comme juste lorsqu'il se formait sous l'empire de l'offre et de la demande. [...] Le prix était juste même s'il ne laissait pas au producteur un revenu suffisant pour faire vivre sa famille et continuer son exploitation. Une conception plus humaine s'est imposée aujourd'hui. Nous croyons que le prix n'est pas juste s'il ne permet pas à l'agriculteur de vivre décemment. » (Milhau, Montagne, $1968)^{24}$. Ajoutons qu'il serait tout aussi injuste s'il ne permettait au plus grand nombre de se nourrir convenablement.

24. Milhau J., Montagne R. (1968). Économie rurale. Paris, Presses universitaires de France, coll. «Thémis». 